\title{
Effects of Adding Dexpanthenol to Prednisolone in an Experimental Model of Inflammatory Bowel Disease
}

\author{
Seyedeh Mehrnaz Mostatabzadeh ${ }^{1}(\mathbb{D})$, Seyyed Meysam Abtahi Froushani ${ }^{1 *(i D}$
}

1. Dept. of Microbiology, Faculty of Veterinary Medicine, Urmia University, Urmia, Iran

\begin{tabular}{l}
\hline Article Info \\
\hline doi 10.30699/jambs.28.130.230 \\
\hline Received: 2020/05/07; \\
Accepted: 2020/09/16; \\
Published Online: 21 Sep 2020; \\
\hline Use your device to scan and read the \\
article online \\
Q \\
(1)
\end{tabular}

Corresponding Information: Seyyed Meysam Abtahi Froushani, Dept. of Microbiology, Faculty of Veterinary Medicine, Urmia University, Urmia, Iran

E-Mail: sm.abtahi@urmia.ac.ir

\begin{abstract}
Background \& Objective: Inflammatory bowel diseases (IBDs) represent serious chronic auto-inflammatory conditions, affecting the alimentary tract. The beneficial effect of dexpanthenol has been observed on some inflammatory conditions. Here, the therapeutic potential of combined dexpanthenol and prednisolone in alleviating the symptoms of the animal model of IBD was investigated.
\end{abstract}

Materials \& Methods: Luminal instillation of $4 \%$ acetic acid ( $2 \mathrm{ml}$ for each rat) was used to induce IBD. Rats in the treatment groups received dexpanthenol $(500 \mathrm{mg} / \mathrm{kg})$, prednisolone $(2 \mathrm{mg} / \mathrm{kg}$ ), or a combination of both (half doses of each drug) by oral gavage for 11 consecutive days.

Results: Dexpanthenol could regress the clinical scores of the IBD model more than prednisolone. More importantly, combination therapy with half doses of dexpanthenol and prednisolone caused more considerable improvement in the disease activity index (DAI) compared to IBD rats received monotherapy. Both monotherapies promoted a remarkable decrease in the messenger RNA (mRNA) expression of NF- $\mathrm{kBp} 65$, as well as the levels of interleukin 6 (IL-6) and TNF- $\alpha$ activity in the inflamed colon. Dexpanthenol could regress the intensity of malondialdehyde (MDA), myeloperoxidase (MPO), and nitric oxide (NO) in the inflamed colon more than prednisolone. More importantly, our results demonstrated that combination therapy resulted in a much more prominent decrease in the level of TNF- $\alpha$ and NF- $\mathrm{\kappa Bp} 65$, as well as the level of superoxide dismutase (SOD) and total gut protein content, than monotherapy. Conversely, combination therapy resulted in a much more pronounced decrease in NO and MDA levels than those recorded in IBD rats received individual treatment. Finally, the mRNA level of IאB $\alpha$ did not show any remarkable discrepancy between the experimental groups.

Conclusion: The combination of dexpanthenol and prednisolone could be used as a promising strategy to alleviate the signs of IBD.

Keywords: Dexpanthenol, Prednisolone, Inflammatory bowel diseases, Combination therapy

\section{Introduction}

Inflammatory bowel diseases (IBDs) represent serious chronic auto-inflammatory conditions, affecting the alimentary tract in dogs and humans (1). However, the principal etiology of IBD is uncertain, and the progression of the disease is thought to originate as a consequence of aberrant immune responses and continuous inflammation against the microbiome in the gut (2).

Diverse classes of immunomodulatory or antiinflammatory drugs, e.g., steroids, 5 -aminosalicylates, cyclosporine, azathioprine, cyclophosphamide, and chlorambucil, can be administered to control extensive inflammatory reactions in IBD (1-3). Despite the relative effectiveness, these medications have significant side effects, such as infections, indigestion, other alimentary disorders, skin thinning, and visual disorders $(2,3)$. Due to the complexity of autoinflammatory diseases, it does not appear that a monotherapy could be useful in all patients during all periods of IBD. Therefore, it is quite logical to use the combination of new medications or the existing drugs for better IBD control or reduction of the side effects $(4,5)$. Fortunately, the animal model of IBD, e.g., acetic acid-induced colitis in rats, has provided an excellent opportunity to test new drug compounds (3).

Pantothenol (also called panthenol) is biologically active amino alcohol and a monocarboxylic acid amide. Dexpanthenol (D-panthenol, provitamin B5) is the only active form of pantothenol, which is quickly oxidized to pantothenic acid (PA; vitamin B5) in an 
organism $(6,7)$. PA is a fundamental component of coenzyme A, which acts as an essential cofactor in metabolic reactions (8). Interestingly, some studies have documented the antioxidant and antiinflammatory benefits of dexpanthenol $(6,8,9)$. For example, the beneficial effect of dexpanthenol has been observed on testicular and renal ischemic damages, diabetic nephropathy, necrotizing enterocolitis, ulcerative colitis, acute lung injury, and experimentally-induced endometriosis (8-10). Also, dexpanthenol can potentiate wounds healing via moisturizing and skin barrier enhancing. In skin irritations and wounds, Dexpanthenol stimulates not only metabolic activity in skin cells, but also gene expression for optimum wound healing (11).

Nonetheless, there is no or limited information on the potential benefits of combined dexpanthenol and prednisolone on IBD. Accordingly, the present investigation was done to assess the potential benefits of co-administering dexpanthenol and prednisolone.

\section{Materials and Methods}

\section{Reagents}

The enzyme-linked immunosorbent assay (ELISA) kits were prepared from PeproTech (UK). Other reagents were purchased from Sigma-Aldrich (St. Louis, MO, USA).

\section{Animals}

In total, 50 male Wistar albino rats, aged 6-7 weeks, weighing 200-250 g were prepared from Empirical Animal Care Center, Urmia University, Urmia, Iran. The rats were maintained under stable situations (i.e., $25^{\circ} \mathrm{C}$ and a 12-hour dark/light cycle) and rendered water and food ad libitum. Ethical considerations concerning research with the laboratory animals were observed, as stated in the regulations of the Ethics Committee of Faculty of Veterinary Medicine, Urmia University, Urmia, Iran (Ref.IR-UU-AEC-142/DA3).

\section{In-vivo Experimental Design}

IBD was induced by intra-rectal instillation of acetic acid, as described previously (12). Briefly, all the Wistar rats maintained under the fast condition for 36 hr. Next, a pediatric catheter was inserted into the anus of the rats flowing light ether anesthesia in a way that the tip of the catheter was inserted about $8 \mathrm{~cm}$ into the end part of the gut. Afterward, a solution of acetic acid diluted to $4 \%$ (4 mL) was administrated into the lumen of the colon by enema. The healthy group was also instilled with normal saline $0.9 \%$ with a volume of 4 $\mathrm{ml}$. Rats were put in the head-down vertical status for $1 \mathrm{~min}$ to prevent acetic acid leakage.

Rats were randomly appointed within four groups of 10 rats as follows: the healthy group, control colitis rats, colitis rats receiving dexpanthenol $(500 \mathrm{mg} / \mathrm{kg}$ every day, oral gavage), colitis rats receiving prednisolone ( $2 \mathrm{mg} / \mathrm{kg}$ every day, oral gavage), and colitis rats receiving a combination of both dexpanthenol and prednisolone (half doses of each drug, oral gavage). The control colitis rats were treated with an equal volume of PBS. Healthy rats were intrarectally received PBS and treated with an equal volume of PBS with the same schedule of IBD rats. The pharmacotherapy was initiated after the induction of colitis and followed all over the investigation until the $11^{\text {th }}$ day when the animals were sacrificed. Afterward, the gut tissues were harvested for further examination.

\section{Evaluation of Disease Severity}

Clinical signs (rectal bleeding, stool blood, and stool consistency) were monitored every day. The disease severity score was monitored; the sum of the scores of the parameters is shown in Table 1 (13). The sum of the scores of all the criteria was used to calculate the disease activity index (DAI). To monitor the length/weight ratio, the length and weight of the colon between the ileocecal junction and rectum were measured. One of the common features of colonic instillation of acetic acid is the enlargement of the spleen. Therefore, the ratio of the spleen weight/body weight of each rat was calculated as the spleen weight index

Table 1. Scoring system for the evaluation of colitis

\begin{tabular}{cccc} 
Score & Weight loss & Stool consistency & \multicolumn{1}{c}{ Blood feces } \\
0 & Negative & Normal & Negative \\
1 & $1-9 \%$ & Soft & Red \\
2 & $10-19 \%$ & Very Soft & Dark Red \\
\hline 3 & $<20 \%$ & Diarrhea & Black \\
\hline
\end{tabular}

Sum of scores of all criteria was used to calculate DAI

Note. DAI: Disease activity index. 


\section{Preparation of Colonic Homogenate}

First, $10 \mathrm{~cm}$ of distal colon's tissue was dissected and washed with normal saline. The same amount of gut was homogenized at 10 volumes of ice-cold normal saline. Then, homogenates were centrifuged at $1200 \mathrm{~g}$ for $10 \mathrm{~min}$ at $4^{\circ} \mathrm{C}(\mathbf{1 4})$.

\section{Biochemical Analysis}

The activity of $(\mathrm{Cu}-\mathrm{Zn}$ and $\mathrm{Mn})$ superoxide dismutase (SOD; EC 1.15.1.1) was monitored, similar to the procedure qualified by Sun et al. (15). The reality of this assay was the blockage of the reduction of nitroblue tetrazolium by the xanthine-xanthine oxidase system as a superoxide anion $\left(\mathrm{O}_{2} \bullet-\right)$ generator. In this system, one unit of SOD was calculated as the enzyme content promoting $50 \%$ occlusion in the rate of nitroblue tetrazolium reduction. The SOD activity was reported as units per $\mathrm{mg}$ of protein.

The activity of glutathione peroxidase (GPx; EC 1.6.4.2) was calculated by the procedure described by Paglia and Valentine (16). In brief, reduced glutathione (GSH), NADPH, and sodium azide were added to a tube. Then, hydrogen peroxide $\left(\mathrm{H}_{2} \mathrm{O}_{2}\right)$ was added to initiate the enzymatic reaction. The shift in absorbance at $340 \mathrm{~nm}$ was spectrophotometrically counted. The activity of GPx was presented as units per $\mathrm{g}$ of protein.

Malondialdehyde (MDA) evaluation was followed, similar to the scheme explained earlier (17). In a short, $2.5 \mathrm{~mL}$ of reaction buffer $(0.25 \mathrm{M} \mathrm{HCl}, 0.37 \%$ thiobarbituric acid, and 15\% trichloroacetic acid, 1:1:1 ratio) was mixed with $100 \mu \mathrm{L}$ of colon homogenate and heated at $95^{\circ} \mathrm{C}$ for about $1 \mathrm{hr}$. Afterward, the mixture was centrifuged at $4000 \mathrm{~g}$ for $10 \mathrm{~min}$. Eventually, the absorbance of the supernatant was spectrophotometrically monitored at $540 \mathrm{~nm}$. Findings were reported as $\mathrm{nM}$ of $\mathrm{MDA} / \mathrm{mg}$ protein.

To evaluate myeloperoxidase (MPO) activity, $10 \mu \mathrm{L}$ of homogenized gut sample was coupled with $80 \mu \mathrm{L}$ of $0.75 \mathrm{mM} \mathrm{H}_{2} \mathrm{O}_{2}$ and $110 \mu \mathrm{L}$ of reaction dilution $(2.9$ $\mathrm{mM} 3,3$ ',5,5'-tetramethylbenzidine [TMB] in $14.5 \%$ dimethyl sulfoxide plus $150 \mathrm{mM}$ of sodium phosphate buffer at $\mathrm{pH}$ 5.4). The absorbance was monitored at 450 $\mathrm{nm}$ (reference: $620 \mathrm{~nm}$ ), and samples were maintained for $15 \mathrm{~min}$ at $37^{\circ} \mathrm{C}$. Thereupon, sulfuric acid $(50 \mu \mathrm{L}$ of $2 \mathrm{M})$ was mixed to stop the reaction, and the opticalabsorbance was recorded at $450 \mathrm{~nm}$. As the standard, $10 \mu \mathrm{L}$ of horseradish peroxidase (2.5 and 25 milliunit/mL) was applied. Finally, MPO activity was reported as the discrepancy of the absorbance in accordance with the standard curve of horseradish peroxidase. Data were presented in milliunits per milliliter (mU/mL) (3).

The Griess method was used to monitor nitric oxide (NO) levels in colonic tissues. Shortly, $100 \mu \mathrm{L}$ of Griess reagent $(0.1 \%$ naphthyl ethylenediamine, $3 \%$ phosphoric acid, and $0.1 \%$ sulphanilamide) was admixed with $100 \mu \mathrm{L}$ of the homogenized colonic specimen. The mixture was kept in the dark at $25^{\circ} \mathrm{C}$ for
10 min. Finally, the absorbance was spectrophotometrically recorded at $540 \mathrm{~nm}$. The nitrite level was reported in accordance with the standard curve (18).

The Lowry protein assay was used to assess the total protein concentration in the gut homogenate.

\section{Evaluation of Inflammatory Cytokines in Colonic Homogenate}

The production intensity of TNF- $\alpha$ and interleukin 6 (IL-6) in the gut samples was calculated by using a commercially available enzyme-linked immunesorbent assay (ELISA) according to the manufacturer's guidelines.

\section{Expression of mRNA of IкB $\alpha$ and NF- $\mathrm{B}$ p65 in the Gut Samples}

The content of messenger RNA (mRNA) in the hind part of colonic tissue was drawn out by a standard kit according to the producer's guidelines. In short, a realtime reverse transcription-polymerase chain reaction (RT-PCR) procedure was applied after synthesizing complementary DNA (cDNA). The $\beta$-actin gene was employed for normalizing mRNA expression. The sense and anti-sense primers are as follows: PCR forward set for $\mathrm{NF}-\kappa \mathrm{B}$ p65 :5'TGCAGGCTCCTGTGCGAGTG-3'; PCR reverse set for NF- $\kappa$ B p 65: 5'-TCCGGTGGCGATCGTCTGTGT-

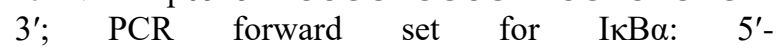
CGTGTCTGCACCTAGCCTCTATC-3'; PCR reverse set for I $\kappa \mathrm{B} \alpha$ : 5'-GCGAAACCAGGTCAGGATTC-3'; PCR forward set for $\beta$-actin: $5^{\prime}$ GCAGGAGTACGATGAGTCCG-3'; and PCR reverse set for $\beta$-actin: $5^{\prime}$ ACGCAGCTCAGTAACAGT CC-3' (13).

\section{Statistical Surveys}

DAI was evaluated by using the non-parametric Kruskal-Wallis exam confirmed by the MannWhitney U evaluation with the Bonferroni adjustment. The rest of the data were examined by one-way analysis of variance (ANOVA) plus Dunnett's posthoc test. Data were represented as means $\pm \mathrm{SD}$. The Pvalues of under 0.05 were assumed statistically significant.

\section{Results}

\section{Monitoring the Disease Activity}

The well-being and weight change of the animals were recorded daily after the instillation of acetic acid into the gut lumen. As shown in Figure 1, there was no significant difference between the outcomes of IBD rats received dexpanthenol or prednisolone at the end of the study. However, regarding the average mean cumulative disease score, the reduction in IBD animals received dexpanthenol was significantly more prominent than in IBD rats treated with prednisolone (Figure 2A). More importantly, combined dexpanthenol and prednisolone in half doses caused 
more considerable improvement in the cumulative disease score compared to IBD rats received both monotherapies (Figure 1).

The statistical analysis of clinical scores indicated that, compared to the IBD group, combination therapy and treatment with dexpanthenol led to a significant reduction in the cumulative disease score from the fourth day after the induction of the disease (Figure 1). However, at the end of the study, the average mean cumulative disease score was considerably lessened in IBD animals treated with combination therapy compared with the dexpanthenol-treated IBD group (Figure 2A). For IBD rats treated with prednisolone, this period was repeated from day 8 after the disease induction (Figure 1).

The ratio of colon weight/length was monitored to evaluate the condition of colonic mucosal damage. The increased weight/length ratio was observed with a significant difference in colitis rats compared to healthy rats (Figure 2B). Treatment with dexpanthenol showed a non-significant decrease in the colon weight/length ratio compared to the treatment with prednisolone (Figure 2B). However, combination therapy showed a significantly lower weight/length ratio than both monotherapies (Figure 2B).

The spleen weight index was increased in the rats with colitis compared to the healthy ones, while all the therapies significantly reduced it (Figure 2C). Treatment with prednisolone promoted a notable decrease in the spleen weight index compared to the treatment with dexpanthenol (Figure 2C). Combination therapy with dexpanthenol and prednosolone led to a remarkable decrease in the spleen weight index compared to the IBD rats received both monotherapies, so that there was no difference in this index between combined treated colitis rats and healthy ones (Figure $2 \mathrm{C})$.

\section{Evaluation of Biochemical Parameters}

The attained data showed marked promotion in MDA content and reduction in GSH content, and SOD activity was noticed in the gut tissues of the colitis rats, when compared with healthy rats (Table 2). Our results indicated that the MDA content in dexpanthenol or combined IBD treated rats was more pronounced than in IBD rats treated with prednisolone. Conversely, dexpanthenol or combination therapy could mount the levels of SOD and GSH more profoundly than prednisolone in IBD rat (Table 2). Also, the obtained data demonstrated that combination therapy resulted in a much more pronounced decrease in MDA levels and a more profound increase in SOD levels than the levels recorded in IBD rats received individual treatment (Table 2).

NO activity, as well as MPO in the gut homogenate of animals with colitis, was markedly mounted compared to healthy rats (Figures $3 \mathrm{~A}$ and $3 \mathrm{~B}$ ). Treatment with dexpanthenol promoted marked regression in MPO activity and the level of NO output in the colonic homogenate of rats with colitis compared with prednisolone-treated IBD rats (Figures 3A and 3B). More importantly, combination therapy resulted in a much more profound decrease in NO than NO levels recorded in IBD rats treated with each medication alone (Figures $3 \mathrm{~A}$ and $3 \mathrm{~B}$ ).

As shown in Figure 3C, a significant decrease in protein concentration in gut homogenates was observed in colitis rats compared to healthy ones. Both monotherapies could reverse the decreasing pattern of protein content in gut tissue without any significant difference (Figure 3C). Combination therapy caused more considerable improvement in the protein content of colonic tissues compared to both monotherapies (Figure 3C).

\section{Monitoring the Level of Pro-inflammatory Cytokines}

As shown in Figure 3, the levels of IL-6 and TNF- $\alpha$ in the gut specimens were significantly increased in vehicle-treated colitis rats compared to healthy animals. All the therapies caused a significant and marked reduction in the content of these cytokines in the gut homogenate of the animals with colitis (Figure 4). Furthermore, our data demonstrated that combination therapy resulted in a much more noticeable decrease in TNF- $\alpha$ level than monotherapy (Figure 4).

Table 1. Scoring system for the evaluation of colitis

\begin{tabular}{|c|c|c|c|}
\hline & MDA (mM/mg) & SOD (U/mg protein) & GSH $(\mu \mathrm{mol} / \mathrm{g})$ \\
\hline Healthy & $16.07 \pm 3.01$ & $0.9 \pm 0.07$ & $2.89 \pm 0.22$ \\
\hline Colitis & $66.54 \pm 6.38^{\$}$ & $0.41 \pm 0.03^{\$}$ & $1.76 \pm 0.13^{\$}$ \\
\hline Colitis+Dexp. & $28.48 \pm 4.27^{\$^{*}}$ & $0.76 \pm 0.05^{\$^{*}}$ & $2.49 \pm 0.19^{\$ *}$ \\
\hline Colitis+Pred. & $44.86 \pm 3.97^{\$ * \&}$ & $0.61 \pm 0.05^{\$ * \&}$ & $2.1 \pm 0.15^{\$ * \&}$ \\
\hline Colitis+Com. & $22.78 \pm 3.81^{* \& \#}$ & $0.83 \pm 0.06 * \& @$ & $2.53 \pm 0.19^{\$ * @}$ \\
\hline
\end{tabular}

Data were reported as mean \pm SD $(\$ P<0.05$ versus healthy rats; $* P<0.05$ versus PBS-treated IBD rats; $\& P<0.05$ versus dexpanthenol-treated RA rats; @ $P<0.05$ versus prednisolone-treated rats).

Note. PBS:Phosphate-buffered saline Dexp: Dexpanthenol-treated IBD group.
IBD: Inflammatory bowel disease.

Pred: Prednisolone-treated IBD group.
RA: Rheumatoid arthritis Com: Combination-treated IBD group. 


\section{Results of RT-PCR Analysis}

As expected, the expression of NF- $\mathrm{Bp} 65$ (nuclear factor kappa-light-chain-enhancer of activated $\mathrm{B}$ cells) mRNA in the hindgut samples was mounted in the animals intrarectally received acetic acid compared to healthy animals (Figure 5A). Both treatments led to a significant reduction in the mRNA level of NF- $\kappa$ Bp65. However, combination therapy caused a more considerable reduction (Figure 5A). Besides, the mRNA level of $\mathrm{I} \kappa \mathrm{B} \alpha$ (inhibitor of $\mathrm{NF}-\kappa \mathrm{B}$ ) showed no statistically significant discrepancy between the experimental groups (Figure 5B).

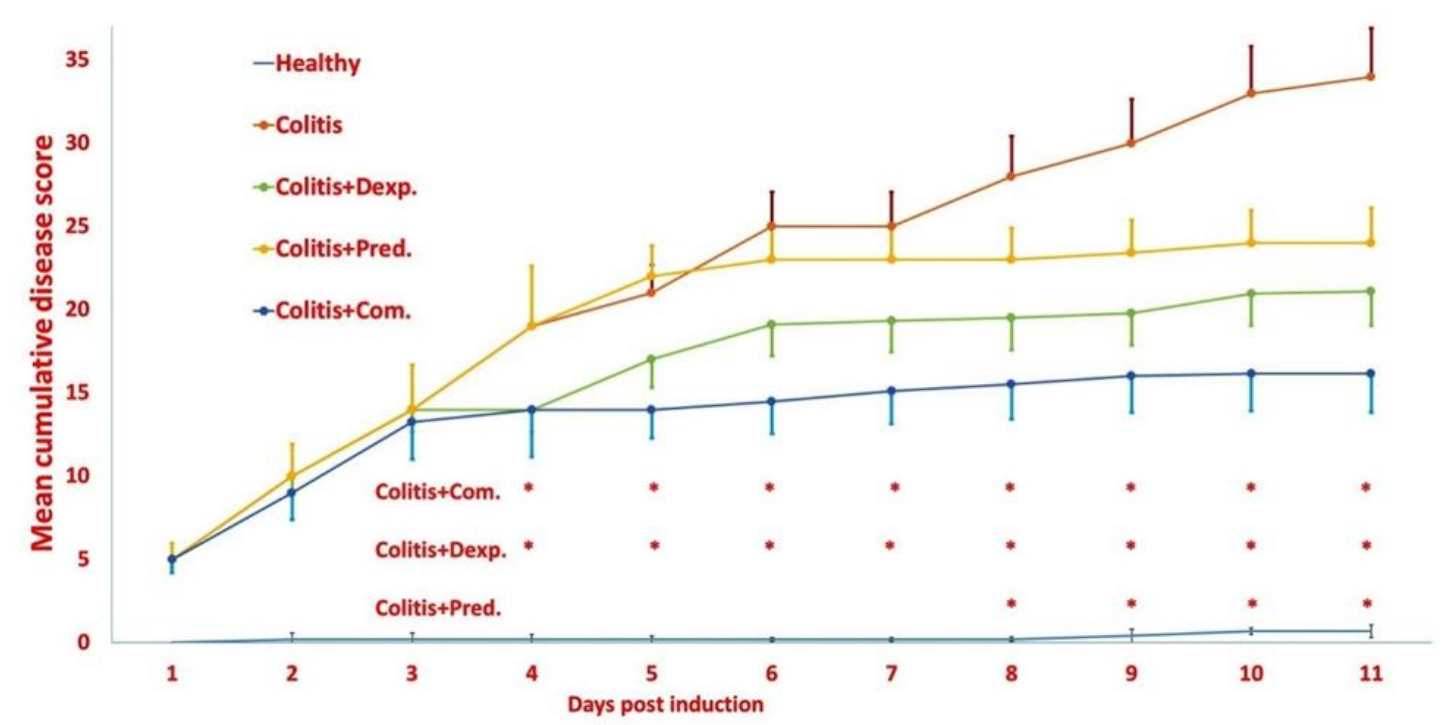

Figure 1. The assessment of the mean cumulative disease score in IBD rats. IBD rats were treated with dexpanthenol and prednisolone alone and in combination. The combination of half doses of dexpanthenol and prednisolone improved clinical outcomes of colitis better than the treatment with either drug alone in full doses. Results are presented as mean $\pm \mathrm{SD}(\$ \mathrm{P}<0.05$ versus healthy rats; $* \mathrm{P}<0.05$ versus $\mathrm{PBS}$-treated IBD rats; $\& \mathrm{P}<0.05$ versus dexpanthenol-treated RA rats; @ $<<0.05$ versus prednisolone-treated rats).

Note. PBS:Phosphate-buffered saline Dexp: Dexpanthenol-treated IBD group.
IBD: Inflammatory bowel disease. Pred: Prednisolone-treated IBD group.
RA: Rheumatoid arthritis Com: Combination-treated IBD group.

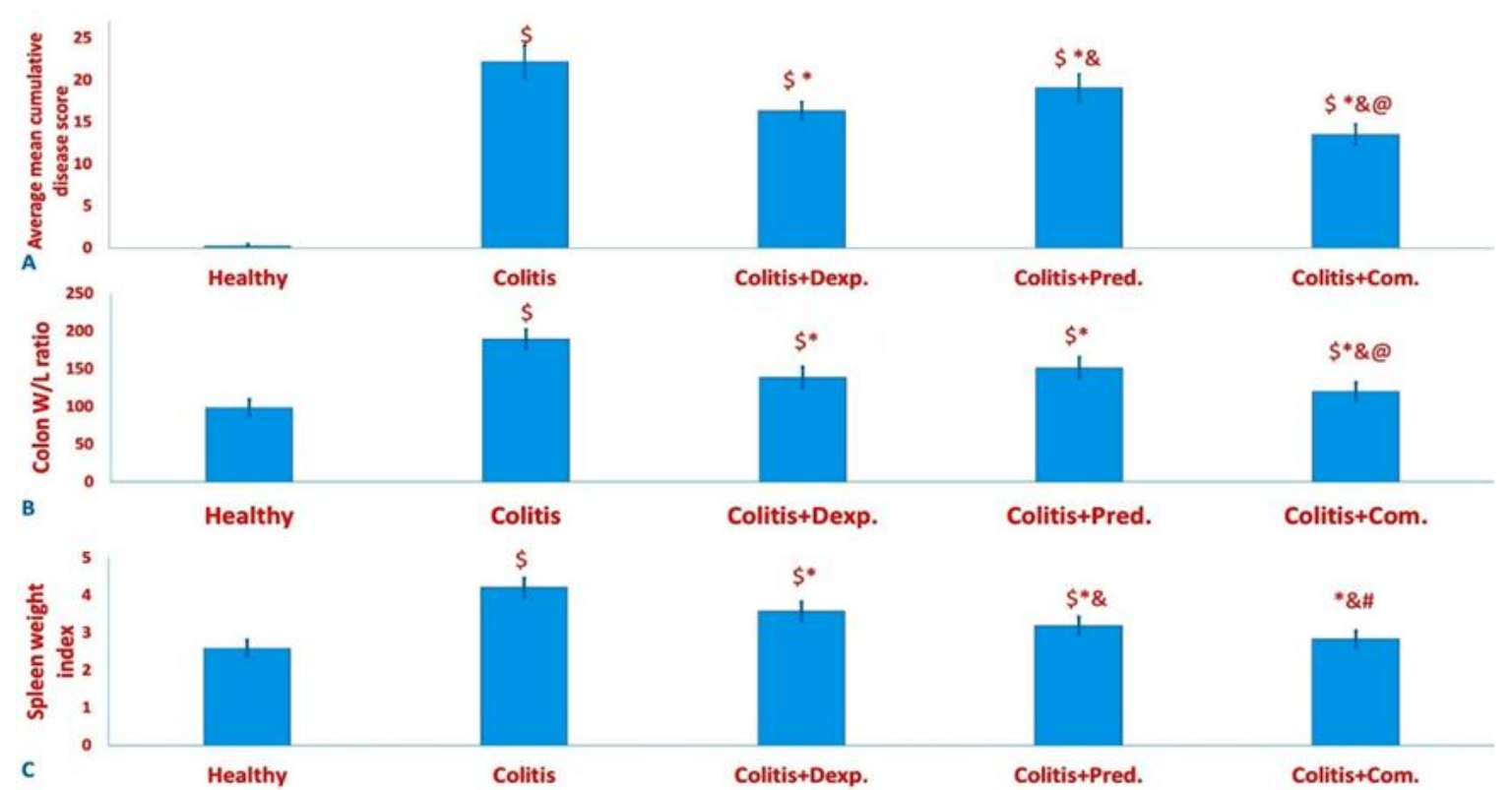

Figure 2. The assessment of clinical features in IBD rats. Results are presented as mean \pm SD $(\$ P<0.05$ versus healthy rats; $* P<0.05$ versus PBS-treated IBD rats; $\& P<0.05$ versus dexpanthenol-treated RA rats; @ $P<0.05$ versus prednisolone-treated rats).

Note. PBS:Phosphate-buffered saline Dexp: Dexpanthenol-treated IBD group.
IBD: Inflammatory bowel disease. Pred: Prednisolone-treated IBD group.
RA: Rheumatoid arthritis Com: Combination-treated IBD group. 


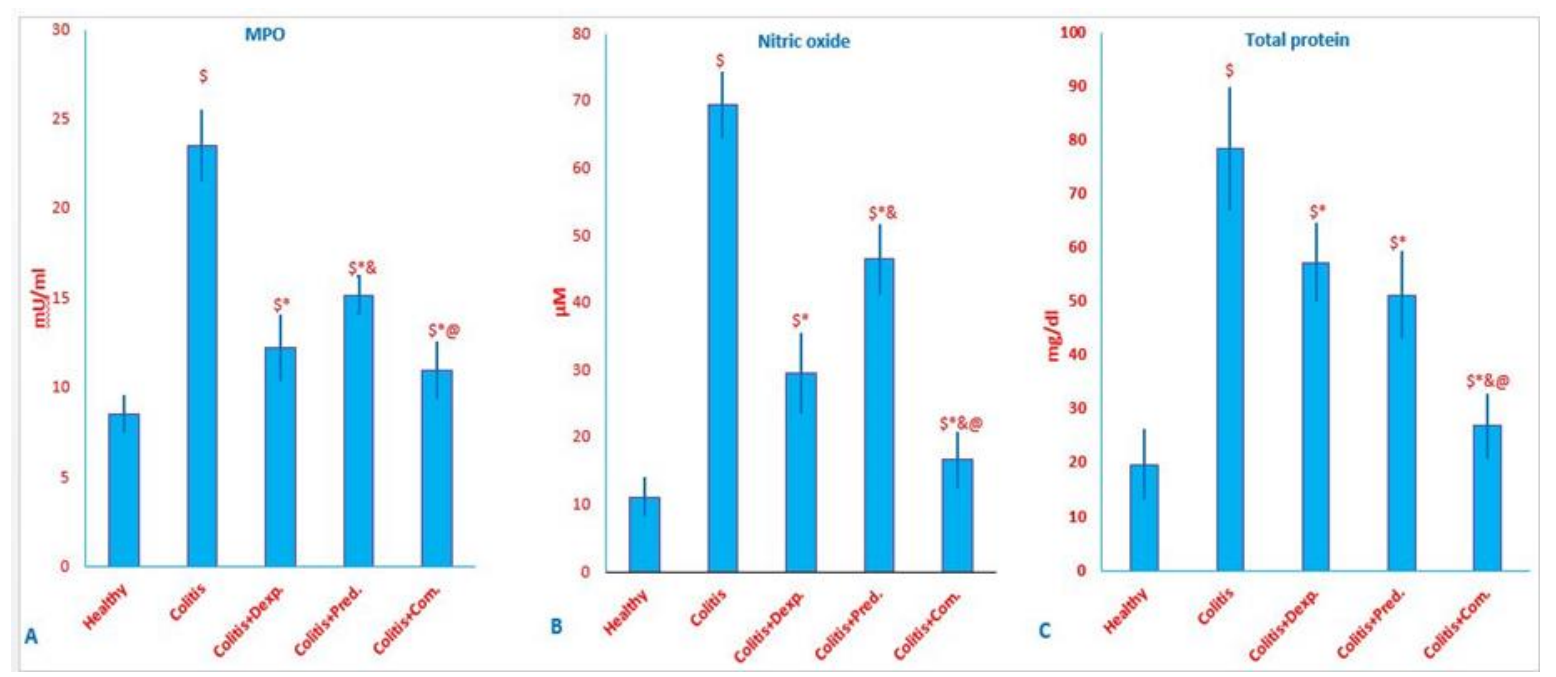

Figure 3. Alteration in some biochemical parameters in the colonic homogenate of colitis rats. Data are reported as mean \pm SD $(\$ P<0.05$ versus healthy rats; $* P<0.05$ versus PBS-treated IBD rats; $\& P<0.05$ versus dexpanthenol-treated RA rats; @ $P<0.05$ versus prednisolone-treated rats).

Note. PBS:Phosphate-buffered saline Dexp: Dexpanthenol-treated IBD group.
IBD: Inflammatory bowel disease. Pred: Prednisolone-treated IBD group.
RA: Rheumatoid arthritis Com: Combination-treated IBD group.

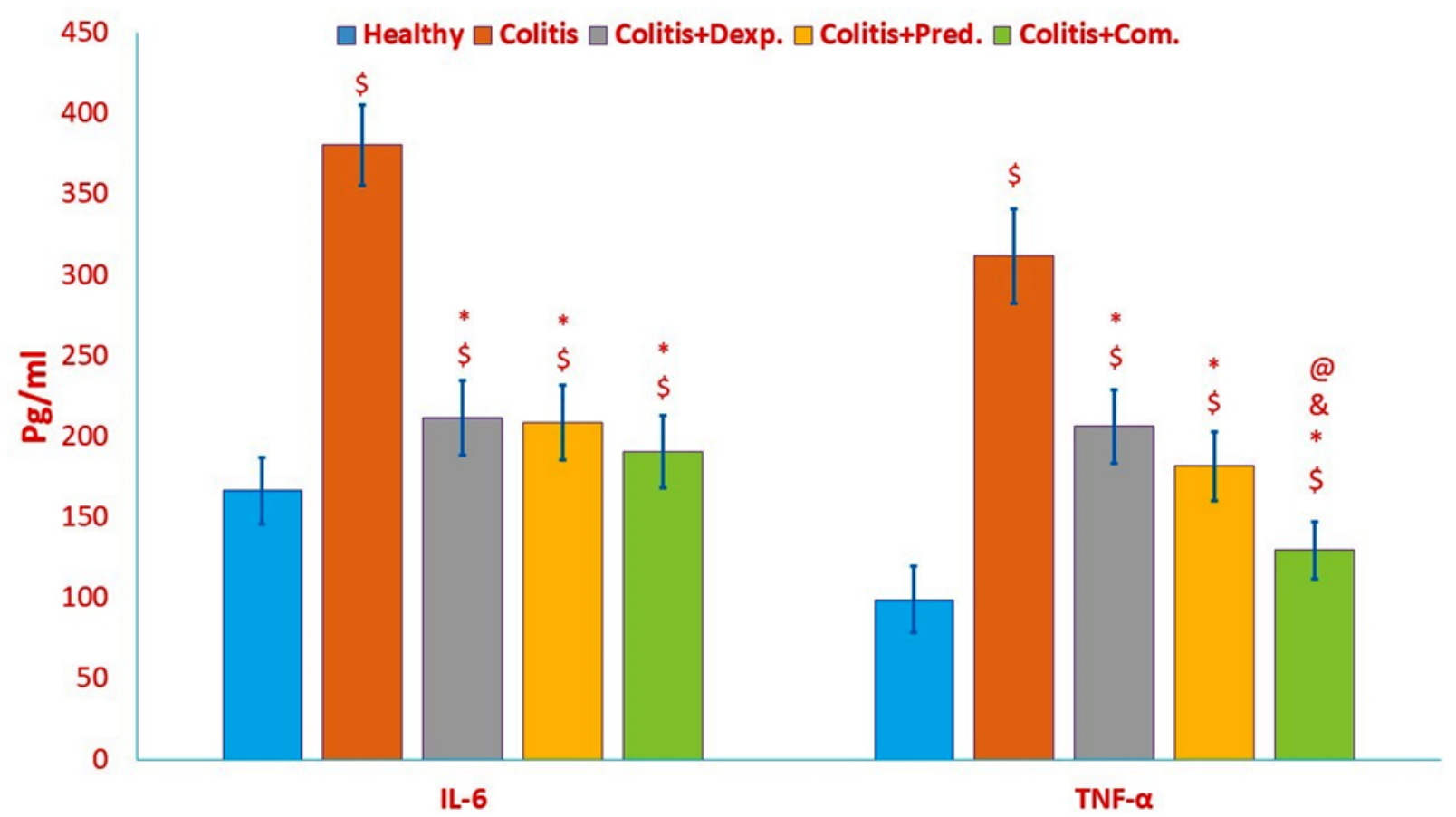

Figure 4. The effect of therapeutic approaches on the levels of pro-inflammatory cytokines in the colonic homogenate of experimental rats. The findings are shown as mean $\pm \mathrm{SD}(\$ P<0.05$ versus healthy rats; $* P<0.05$ versus PBS-treated IBD rats; $\& P<0.05$ versus dexpanthenol-treated RA rats; @ $P<0.05$ versus prednisolone-treated rats).

Note. IL-6: Interleukin 6. TNF- $\alpha$ : Tumor Necrosis Factor Alpha PBS: Phosphate-buffered saline IBD: Inflammatory bowel disease. RA: Rheumatoid arthritis Dexp: Dexpanthenol-treated IBD group. Pred: Prednisolone-treated IBD group. Com: Combination-treated IBD group. 

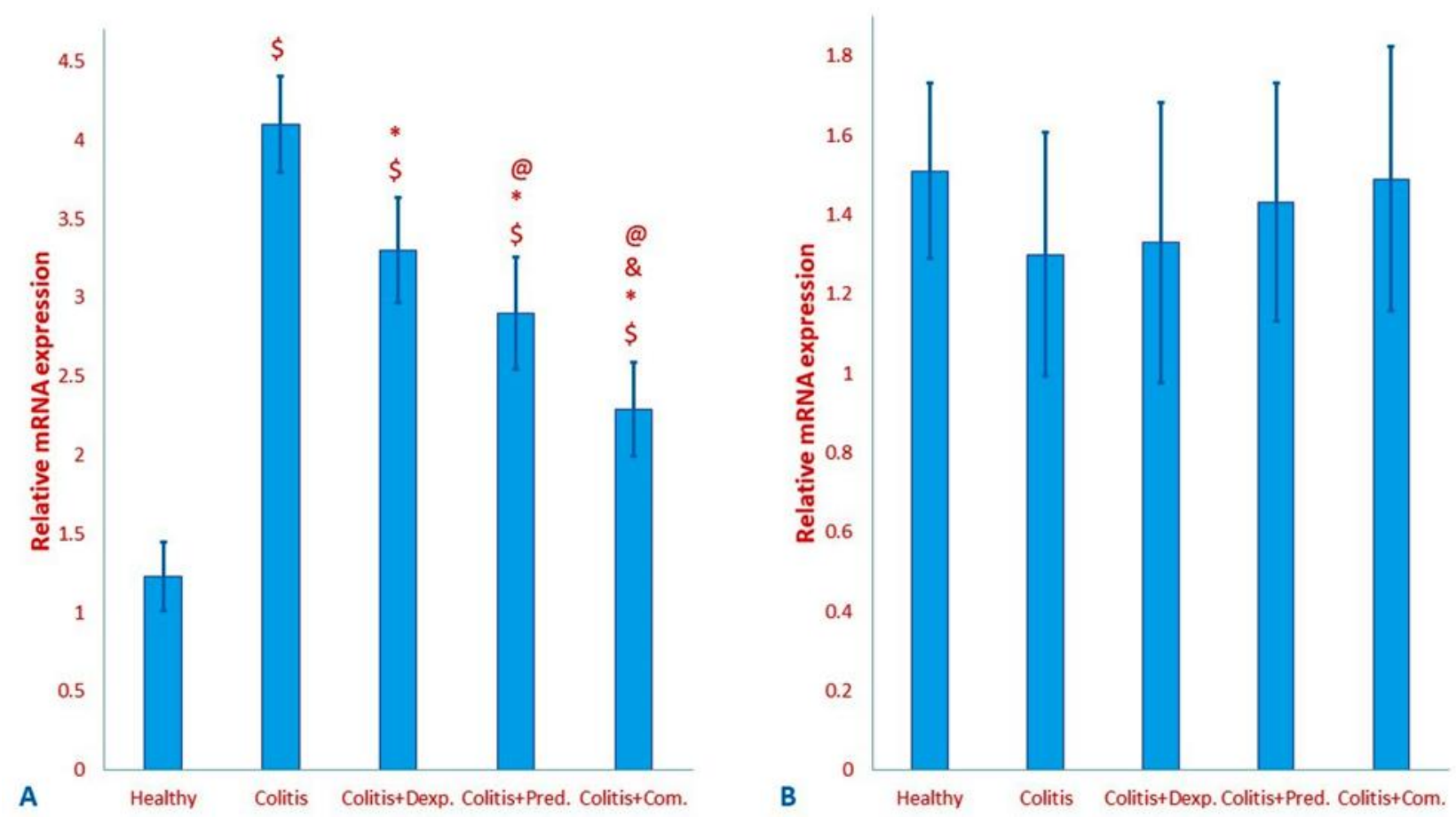

Figure 5. The relative expression of the mRNA of NF- $\kappa$ Bp65 (A) and I $\mathrm{B}(\mathrm{B})$ in the colonic specimen. Results are presented as mean \pm SD $(\$ P<0.05$ versus healthy rats; $* P<0.05$ versus PBS-treated IBD rats; $\& P<0.05$ versus dexpanthenol-treated RA rats; @ $P<0.05$ versus prednisolone-treated rats).

Note. mRNA: Messenger RNA. NF-кBp65: p65 subunit of nuclear factor kappa-light-chain-enhancer of activated

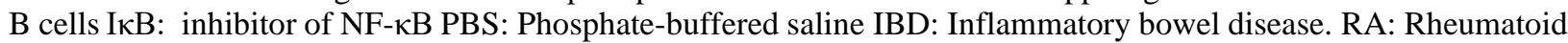
arthritis Dexp: Dexpanthenol-treated IBD group. Pred: Prednisolone-treated IBD group. Com: Combination-treated IBD group.

\section{Discussion}

Combination therapy, along with a different antiinflammatory mechanism for controlling the complicated situation induced by immunological disorder, is a logical decision $(19,20)$. Dexpanthenol is an alcohol with biological activity analog of PA, which transforms into PA within cells (6). PA performs its anti-oxidative benefits by augmenting the synthesis of reduced GSH and related peroxidase enzymes, which render as the utmost protective machinery versus oxidative stress and lipid peroxidation in the inflammation PA performs its anti-oxidative benefits by augmenting the synthesis of reduced GSH and related peroxidase enzymes (8). Moreover, PA merges into the infrastructure of coenzyme A, promotes anti-inflammatory benefits, and exerts epithelization $(7,8)$. On the other hand, prednisolone is a famous glucocorticoid, which up-regulates the expression of anti-inflammatory proteins and, simultaneously, downregulates the expression of pro-inflammatory proteins (21).

The main propose of combination therapy is to promote significant regression in the clinical outcomes, which is superior to pharmacotherapy with each drug alone (20). The findings of the current investigation suggested that the combined dexpanthenol and prednisolone in half doses led to a remarkable decrease in the adversity of IBD, which was more pronounced than each medication alone. The current study also indicated that combination therapy could enforce guthealing because combined medications can turn out the severity of diminution in total protein levels.

Moreover, it is necessary that each medication prescribed for combination therapy possesses a good safety margin and does not induce further toxicities when used simultaneously $(19,20)$. In this investigation, no bright adverse incident was reported in experimental groups. Also, combined therapy increased weight-gaining in rats.

According to IBD pathogenesis, just after the initial mucosal damage in the gut, inflammatory cells produced a large number of reactive oxygen and nitrative substances, such as $\mathrm{H} 2 \mathrm{O} 2$, hydroxyl radical, superoxide, NO, and peroxynitrite (22). MDA is a good predictor of lipid peroxidation (13). MPO enzyme is a peroxidase that catalyzes the production of hypochlorous (or hypobromous) acid and oxidizes tyrosine to the tyrosyl radical by $\mathrm{H}_{2} \mathrm{O}_{2}$.

Both tyrosyl radical and hypochlorous acid are potentially cytotoxic, so that they may induce oxidative 
damage in the host tissue $(13,23)$. Tissues exposed to inflammation defend against reactive nitrogen species and reactive oxygen species via endogenous enzymatic antioxidants (such as SOD) and endogenous nonenzymatic antioxidants (such as GSH) (24). Previous documents have also indicated that dexpanthenol markedly attenuates MDA contents and MPO activity and also mounts GSH and SOD activity in the LPSchallenged lung tissue and cisplatin-induced hepatotoxicity model $(6,9)$.

Although the infiltration of inflammatory cells could be controlled relatively by corticosteroids, tissue oxidative and nitrative damages are not directly influenced by these agents $(13,23)$. It has been documented that dexpanthenol possesses antioxidative benefits via enhancing the expression of reduced GSH and its related peroxidase enzymes (25). Our results indicated that the intensity of MDA, MPO, and NO was suppressed in dexpanthenol or combined IBD treated rats more profoundly than in IBD rats received prednisolone. Conversely, dexpanthenol or combination therapy could increase the levels of SOD and GSH more profoundly than prednisolone in IBD rats.

It shall be noted that MPO can be applied as an indirect indicator to assess the severity of neutrophil infiltration into inflamed tissue, such as a colon in IBD condition (3). As described previously, dexpanthenol can accelerate re-epithelization and promote wound healing (8). Also, damage to the mucosal cell and loss of mucosal cell integrity is the first stage in the development of IBD, such as acetic acid-induced colitis $(3,13,23)$. Therefore, a part of the benefit observed in combination therapy may be due to the potent anti-inflammatory effect of prednisolone together with the antioxidant properties and epithelial repair features of dexpanthenol. In this regard, the results indicated that combination therapy led to a much more pronounced decrease in NO and MDA levels and also more profoundly increased the level of SOD than the levels recorded in IBD rats received individual treatment.

IL-6 and TNF- $\alpha$ have vital roles in the pathology of IBD $(22,26)$. Medication with the biological inhibitors of TNF- $\alpha$ substantially regresses the signs of IBD (27). TNF- $\alpha$ also participates in pyrexia, algesia, and cachexia (26). IL-6 promotes the production of acutephase proteins, such as C-reactive protein (CRP) (28). $\mathrm{NF}-\kappa \mathrm{B}$, as a master vigorous inflammatory transcription factor, can promote the secretion of proinflammatory mediators, such as IL-6 and TNF- $\alpha$ (13). Recently, the NF- $\kappa \mathrm{B}$ pathway and their downstream signal regulators, including IL- 6 and TNF- $\alpha$, have been considered as new promising anti-inflammatory strategies for controlling IBD (29).

The findings of this survey established that both monotherapies led to a remarkable reduction in the mRNA expression of NF- $\kappa \mathrm{Bp} 65$, production intensity of IL- 6 , and TNF- $\alpha$ in the inflamed colon. Previous data have also shown that TNF- $\alpha$ and IL-6 in the fluid of the bronchoalveolar lavage of mice with acute lung injury were decreased by dexpanthenol administration (6). TNF- $\alpha$ levels were also significantly decreased in a rat endometriosis model treated with dexpanthenol compared to the healthy group (7). More importantly, our results demonstrated that combination therapy resulted in a much more prominent reduction in the levels of TNF- $\alpha$ and NF- $\kappa$ Bp65 than both monotherapies. A further decrease in the level of TNF$\alpha$ may be the reason for the better weight gain in the combination therapy group. I $\kappa \mathrm{B} \alpha$ can suppress NF- $\kappa \mathrm{B}$ signaling by blocking nuclear localization signals (30). The obtained data of this study indicated that the mRNA level of $\mathrm{I} \kappa \mathrm{B} \alpha$ did not show any significant diversity between the experimental groups.

Collectively, this research represented for the first time that treatment with dexpanthenol could subtract the clinical schema of the animal model of IBD more than prednisolone. Our data also indicated for the first time that combined therapy with a half dose of dexpanthenol and prednisolone could alleviate the clinical and laboratory indices of the animal model of IBD more significant than the treatment with each drug alone. Combination therapy promoted a reduction in the levels of oxidative and nitrative stress marker concurrent with the levels of TNF- $\alpha$ and NF- $\kappa$ Bp65 more impressively than each therapy alone.

Accordingly, a combination of these medications can be suggested as a potential strategy for better IBD management. Nevertheless, the present study was only a preliminary investigation conducted in only one animal model. Gut inflammation induced by Acetic acid were most similar to the ulcerative colitis form of human IBD. Hence, further research is required to recognize the accurate mechanism of the reported synergistic benefit between dexpanthenol and prednisolone. Also, if the results of the research on other animal models are appropriate, it is necessary to begin the initial phase of clinical studies in humans.

\section{Acknowledgments}

The authors would like to appreciate all the contributors to the present survey.

\section{Conflict of Interest}

Authors declared no conflict of interest.

\section{References}

1. Cerquetella M, Spaterna A, Laus F, et al. Inflammatory bowel disease in the dog: differences and similarities with humans. World J Gastroenterology. 2010;16(9):1050-6. [DOI:10.3748/wjg.v16.i9.1050]

2. Grosse CSJ, Christophersen CT, Devine A, Lawrance IC. The role of a plant-based diet in 
the pathogenesis, etiology and management of the inflammatory bowel diseases. Expert Rev Gastroenterol Hepatology. 2020;3:1-9. [DOI:10.1080/17474124.2020.1733413]

3. Abtahi Froushani SM, Mashhouri S. The effect of mesenchymal stem cells pulsed with 17 betaestradiol in an ameliorating rat model of ulcerative colitis. Zahedan J Res Med Sci. 2019;21(4):e83762. [DOI:10.5812/zjrms.83762]

4. Agrawal G, Clancy A, Sharma R, Huynh R, Ramrakha S, Borody T. Targeted combination antibiotic therapy induces remission in treatment-naive crohn's disease: A case series. Microorganisms. $2020 ; 8(3)$. [DOI:10.3390/microorganisms8030371]

5. Yousefi-Ahmadipour A, Ebrahimi-Barough S, Niknia S,et al. Therapeutic effects of combination of platelet lysate and sulfasalazine administration in TNBS-induced colitis in rat. Biomed Pharmacother. 2020;125:109949. [DOI:10.1016/i.biopha.2020.109949]

6. Li-Mei W, Jie T, Shan-He W, Dong-Mei M, Peng-Jiu Y. Anti-inflammatory and antioxidative effects of dexpanthenol on lipopolysaccharide induced acute lung injury in mice. Inflammation. 2016;39(5):1757-63. [DOI:10.1007/s10753-016-0410-7]

7. Soylu Karapinar O, Pinar N, Ozgur T, et al. The protective role of dexpanthenol on the endometrial implants in an experimentally induced at endometriosis model. Reproduc Sci (Thousand Oaks, Calif). 2017;24(2):285-90. [DOI: $10.1177 / 1933719116653682]$

8. Gheita AA, Gheita TA, Kenawy SA. The potential role of B5: A stitch in time and switch in cytokine. Phytother Res. 2020;34(2):306-14. [DOI:10.1002/ptr.6537]

9. Karadag A, Ozdemir R, Kurt A, et al. Protective effects of dexpanthenol in an experimental model of necrotizing enterocolitis. J Pediatr Surg. 2015;50(7):1119-24. [DOI:10.1016/i.jpedsurg.2014.10.053]

10. Etensel B, Ozkisacik S, Ozkara E, et al. Dexpanthenol attenuates lipid peroxidation and testicular damage at experimental ischemia and reperfusion injury. Pediatr Surg Int. 2007;23(2):177-81. [DOI:10.1007/s00383-0061781-X]

11. Baron JM, Glatz M, Proksch E. Optimal support of wound healing: New Insights. Dermatol (Basel, Switzerland). 2020:1-8 [DOI:10.1159/000505291]

12. Low D, Nguyen DD, Mizoguchi E. Animal models of ulcerative colitis and their application in drug research. Drug Des Develop Ther. 2013;7:1341-57. [DOI:10.2147/DDDT.S40107]
13. Tahmasebi P, Abtahi froushani SM, Afzale Ahangaran N. Thymol has beneficial effects on the experimental model of ulcerative colitis. Avicenna J Phytomed. 2019;9(6):538-50.

14. Al-Rejaie SS, Abuohashish HM, Al-Enazi MM, Al-Assaf AH, Parmar MY, Ahmed MM. Protective effect of naringenin on acetic acidinduced ulcerative colitis in rats. World $\mathrm{J}$ Gastroenterol. 2013;19(34):5633-44. [DOI:10.3748/wig.v19.i34.5633]

15. Sun Y, Oberley LW, Li Y. A simple method for clinical assay of superoxide dismutase. Clin Chem. 1988;34(3):497-500. [DOI:10.1093/clinchem/34.3.497]

16. Paglia DE, Valentine WN. Studies on the quantitative and qualitative characterization of erythrocyte glutathione peroxidase. J Lab Clin Med. 1967;70(1):158-69.

17. Al-Rejaie SS, Aleisa AM, Sayed-Ahmed MM, et al. Protective effect of rutin on the antioxidant genes expression in hypercholestrolemic male wistar rat. BMC Complement Alternat Med. 2013;13:136. [DOI:10.1186/1472-6882-13-136]

18. Bryan NS, Grisham MB. Methods to detect nitric oxide and its metabolites in biological samples. Free Rad Biol Med. 2007;43(5):645-57. [DOI:10.1016/j.freeradbiomed.2007.04.026]

19. Abtahi Froushani SM, Delirezh N, Hobbenaghi R, Mosayebi G. Synergistic effects of atorvastatin and all-trans retinoic acid in ameliorating animal model of multiple sclerosis. Immunol Invest. 2014;43(1):54-68. [DOI:10.3109/08820139.2013.825269]

20. Golbahari S, Abtahi Froushani SM. Synergistic benefits of nicotine and thymol in alleviating experimental rheumatoid arthritis. Life Sci. 2019;239:117037. [DOI:10.1016/j.lfs.2019.117037]

21. Stahn C, Lowenberg M, Hommes DW, Buttgereit F. Molecular mechanisms of glucocorticoid action and selective glucocorticoid receptor agonists. Molec Cell Endocrinol. 2007;275(1-2):71-8. [DOI:10.1016/j.mce.2007.05.019]

22. Zhang YZ, Li YY. Inflammatory bowel disease: pathogenesis. World J Gastroenterol. 2014;20(1):91-9. [DOI:10.3748/wjg.v20.i1.91]

23. Abtahi Froushani SM, Mashouri S. The beneficial effects of hypiran in Ameliorating Rat Model of Ulcerative Colitis. Zahedan J Res Med Sci. 2018;20(1):e58919. [DOI:10.5812/zjrms.58919]

24. Bilgic Y, Akbulut S, Aksungur Z, et al. Protective effect of dexpanthenol against cisplatin-induced hepatotoxicity. Experiment 
Therap Med. 2018;16(5):4049-57. [DOI:10.3892/etm.2018.6683]

25. Wojtczak L, Slyshenkov VS. Protection by pantothenic acid against apoptosis and cell damage by oxygen free radicals--the role of glutathione. BioFactors (Oxford, England). 2003;17(1-4):61-73.

[DOI:10.1002/biof.5520170107]

26. Owusu G, Obiri DD, Ainooson GK, et al. Acetic acid-induced ulcerative colitis in sprague dawley rats is suppressed by hydroethanolic extract of Cordia vignei Leaves through reduced serum levels of TNF-alpha and IL-6. Int J Chron Dis. 2020;2020:8785497. [DOI:10.1155/2020/8785497]

27. Verstockt B, Ferrante M, Vermeire S, Van Assche G. New treatment options for inflammatory bowel diseases. J Gastroenterol. 2018;53(5):585-90. [DOI:10.1007/s00535-0181449-z]
28. Gautam MK, Goel S, Ghatule RR, Singh A, Nath G, Goel RK. Curative effect of Terminalia chebula extract on acetic acid-induced experimental colitis: role of antioxidants, free radicals and acute inflammatory marker. Inflammopharmacol. 2013;21(5):377-83. [DOI:10.1007/s10787-012-0147-3]

29. Neurath MF. Cytokines in inflammatory bowel disease. Nature Rev Immunol. 2014;14(5):32942. [DOI:10.1038/nri3661]

30. Li Z, Zheng J, Zhang N, Li C. Berberine improves airway inflammation and inhibits NFkappaB signaling pathway in an ovalbumininduced rat model of asthma. $\mathbf{J}$ Asthma. 2016;53(10):999-1005.

[DOI:10.1080/02770903.2016.1180530]

\section{How to Cite This Article:}

Motatabzadeh S M, Abtahi Froushani S M. Effects of Adding Dexpanthenol to Prednisolone in an Experimental Model of Inflammatory Bowel Disease. J Adv Med Biomed Res. 2020; 28 (130) :237-246

\section{Download citation:

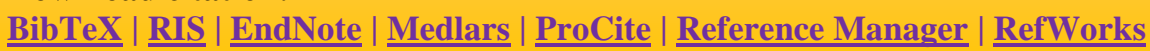

\section{Send citation to:}

8 Mendeley 2 Zotero : 